\title{
BIOINFORMATICS INSPIRED ALGORITHM FOR STEREO CORRESPONDENCE
}

\author{
Romain Dieny, Jerome Thevenon, Jesus Martinez-del-Rincon, Jean-Christophe Nebel \\ Digitial Imaging Research Centre, Kingston University, Kingston-Upon-Thames, KT1 2EE, UK \\ \{R.Dieny, J.Thevenon, Jesus.Martinezdelrincon, J.Nebel\} @kingston.ac.uk
}

\begin{abstract}
Keywords: Stereo correspondence, dynamics programming, sequence alignment, bioinformatics, parameter estimation.
Abstract: $\quad$ In this paper, we exploit the analogy between protein sequence alignment and image pair correspondence to design a bioinformatics-inspired framework for stereo matching based on dynamic programming. This approach also led to the creation of a meaningfulness graph, which helps to predict matching validity according to image overlap and pixel similarity. Finally, we propose an automatic procedure to estimate automatically all matching parameters. This work is evaluated qualitatively and quantitatively using a standard benchmarking dataset and by conducting stereo matching experiments between images captured at different resolutions. Results confirm the validity of the computer vision/bioinformatics analogy to develop a versatile and accurate low complexity stereo matching algorithm.
\end{abstract}

\section{INTRODUCTION}

Stereo matching is an essential step in the process of 3D reconstruction from a pair of stereo images. Since it has many applications including robot navigation, security and entertainment, it has been an important field of computer vision for several decades. The problem of finding correspondences between pixels belonging to a pair of stereo images has been tackled using a wide range of techniques such as block correlations, dynamic programming, graph cut and simulated annealing; excellent reviews of the literature can be found in (Scharstein \& Szeliski 2002) and (Lazaros, et al., 2008). Among these techniques, those based on dynamic programming (DP) have proved particularly attractive. They provide good accuracy and are computationally efficient (MacLean, et al., 2010): they are able to find the global minimum for independent scanlines in polynomial time.

Although the design of some of these DP algorithms was inspired by that of Needleman and Wunsch (1970), e.g. (MacLean, et al., 2010), which was developed for alignment of protein sequences, to our knowledge, no author has exploited fully the analogy between protein and image correspondence. In this paper, we present a DP algorithm for stereo matching inspired by bioinformatics techniques. Not only does the bioinformatics analogy allow the design of an efficient stereo-matching algorithm, but it also permits investigating the limits of applicability of the algorithm in term of image overlap and pixel occlusion. This is illustrated here by producing dense disparity maps from images captured at different resolutions. Finally, we also propose a methodology allowing automatic configuration of all algorithm parameters.

The structure of this paper is organised as follows. After reviewing relevant literature, we detail our novel stereo matching algorithm. Then, experiments are conducted on a benchmark dataset to validate our method. Finally, conclusions and future work are presented.

\subsection{Related Work}

First applications of DP to the problem of stereo matching produced sparse disparity maps using edge information (Baker \& Binford 1981) and (Ohta \& Kanade 1985). In order to generate dense maps, correspondences between scanlines were computed using pixel colour values. This task highlighted complications which were not present when dealing only with edges: they include image noise, indistinct image features and half occlusion, e.g. object points which can be seen only in one of the two images. 
Statistical frameworks have been proposed to explicitly tackle these issues (Geiger, et al., 1992), (Belhumeur, 1996), (Cox, et al., 1996) and (Torr \& Criminisi 2004). Alternatively, (Bobick \& Intille 1999) suggested to pre-process images by producing a 'disparity-space image' based on block correlations and, then, use DP to find the optimal correspondences. In addition to rely on additional free parameters, all these approaches required additional calculations, which affect significantly the computational complexity of the stereo matching process.

Since traditional DP algorithms compute linebased global optimisations, they do not take into account vertical consistency between scanlines. Although some early methods attempted to address this issue (Ohta \& Kanade 1985), (Belhumeur, 1996), (Cox, et al., 1996) and (Bobick \& Intille 1999), they only refine results produced from scanline optimisation. In order not to bias optimisation towards one direction, e.g. scanline, a new class of DP algorithms, which can be applied efficiently to tree structures, has been recently proposed (Veksler, 2005) and (Deng \& Lin 2006). Results show they are significantly more accurate than scanline based methods with only a marginal increase of computational cost.

In the last few years, the main emphasis has been on designing real-time solutions by adapting previous DP algorithms (Forstmann, et al., 2004), (Wang, et al., 2006) and (Salmen, et al., 2009). Eventually, the first FPGA hardware implementation of a DP-based stereo matching algorithm has just been proposed (MacLean, et al., 2010). Its performance demonstrates DP-based approaches provide the best compromise between accuracy and speed.

\section{METHODOLOGY}

We propose a new matching algorithm particularly suitable for the scanline to scanline correspondence problem, which can be applied to pairs of rectified stereo images. First, we introduce the bioinformatics technique on which it is based. Then, we explain how it can be extended to image processing.

\section{1 'Needleman-Wunsch' algorithm}

The publication of the first 'Atlas of Protein Sequence and Structure' (Dayhoff, et al., 1965) which comprised the sequences of 65 proteins, arguably funded the field of bioinformatics. This gave researchers the opportunity to compare sequences to establish evolutionary relationship between proteins. Since protein sequences have an average length of 400 characters and mutate through substitution, insertion and deletion of characters, the alignment of a protein pair is not a trivial matter. The 'Needleman-Wunsch' algorithm (Needleman \& Wunsch 1970) has provided an effective automatic method to produce an exact solution to the global alignment of two protein sequences. It is still at the core of the latest search engines (Altschul, et al., 1997) and (Mackey, et al., 2002), which allow finding the best alignment between a given protein sequence and a large database such as UniProt (Leinonen, et al., 2004), which contains more than 20 million entries.

The 'Needleman-Wunsch' (N\&W) algorithm is based on a dynamic programming approach which optimises the global alignment of character strings according to a scoring function taking into account possible mutations. In practice, alignments are produced by, first, filling in a scoring matrix and, then, 'backtracking' from the highest score in either the last column or the last line of the matrix.

Each matrix cell stores the maximum value which can be achieved by extending a previous alignment (see Table 1). This can be done either by aligning the next character of the first sequence with the next character of the second sequence or extending either sequence by an empty character to record a character insertion or deletion ('indel').

In the case of character alignment, i.e. diagonal motion in the matrix, the score depends on their values. A reward, match, is allocated if the two characters are identical, otherwise a penalty, mismatch, is applied since this highlights a mutation (substitution). When a sequence is extended, i.e. from either north or west, this is also penalised, gap, because it reveals that a mutation (insertion or deletion) occurred. While completing the matrix, in addition to the score of each cell, the direction(s) from which the score is coming must be recorded since they are used in the 'backtracking' process.

The scoring matrix, $M$, is initialised by setting the initial score (top left cell) to zero and the first line and column according to cumulated gap penalties. Then, $M$ is filled in using the following pseudo-code:

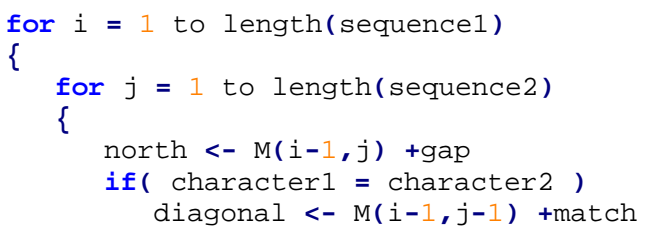




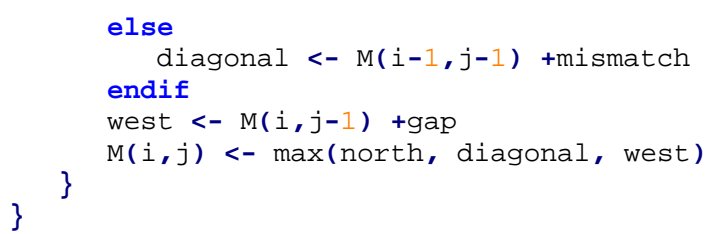

Once the matrix is completed, the optimal alignment is extracted using the 'backtracking' process (see Table 2). First, the highest score cell in either the last column or row is identified. Then, using direction information, a path to the origin of the matrix is constructed. Finally, this path is converted into an alignment. It is important to note that, although the algorithm always finds the best global alignment(s) for a given scoring scheme, there may me several alignments with the optimal score.

The whole process is illustrated with an example in Table 1 and 2, where the following scoring scheme is used: match $=2$, mismatch $=0$ and gap $=-1$. Representing gaps by '-', the resulting alignment is:

$$
\text { EDECE }
$$$$
\mathrm{AD}-\mathrm{CE}
$$

Table 1: Extension of initial alignment. The new cell score is shown in blue; 3 possible scores are shown in green.

\begin{tabular}{|c|c|c|c|c|}
\hline & - & $\mathbf{E}$ & D & $\mathbf{E}$ \\
\hline- & 0 & $\rightarrow-1$ & $\rightarrow-2$ & $\rightarrow-3$ \\
\hline $\mathbf{A}$ & $-1^{\downarrow}$ & ${ }^{\searrow} 0$ & $\stackrel{\searrow}{\rightarrow}-1$ & $\stackrel{\searrow}{\rightarrow}-2$ \\
\hline D & $-2^{\downarrow}$ & ${ }^{\searrow}-1^{\downarrow}$ & ${ }^{\prime} 2$ & ${ }_{1}^{-1} \mathbf{1}^{-3}$ \\
\hline
\end{tabular}

Table 2: Completed scoring matrix and optimal path highlighted in red.

\begin{tabular}{|c|c|c|c|c|c|c|}
\hline & - & $\mathbf{E}$ & D & $\mathbf{E}$ & C & $\mathbf{E}$ \\
\hline- & 0 & $\rightarrow-1$ & $\rightarrow-2$ & $\rightarrow-3$ & $\rightarrow-4$ & $\rightarrow-5$ \\
\hline $\mathbf{A}$ & $-1^{\downarrow}$ & ${ }^{{ }^{\prime}} \mathbf{0}$ & $\stackrel{y}{\rightarrow}-1$ & $\stackrel{y}{\rightarrow}-2$ & $\stackrel{y}{\rightarrow}-3$ & $\stackrel{y}{\rightarrow}-4$ \\
\hline D & $-2^{\downarrow}$ & ${ }^{\searrow}-1^{\downarrow}$ & ${ }^{\searrow} 2$ & $\rightarrow 1$ & $\rightarrow 0$ & $\rightarrow-1$ \\
\hline $\mathrm{C}$ & $-3^{\downarrow}$ & $-2^{\downarrow}$ & $1^{\downarrow}$ & ${ }^{\star} / 2$ & ${ }^{\prime} 3$ & $\rightarrow 2$ \\
\hline $\mathbf{E}$ & $-4^{\downarrow}$ & ${ }^{3}-1$ & $0^{\downarrow}$ & ${ }^{\prime} 3$ & $\stackrel{\searrow}{\rightarrow} 2^{\downarrow}$ & ${ }^{\prime} 5$ \\
\hline
\end{tabular}

\subsection{Application to stereo matching}

An analogy can be made between aligning protein sequences and matching pixels belonging to scanlines, since both tasks aim at establishing optimal correspondence between two strings of characters. In addition, the 'right' image of a stereo pair can be seen as a mutated version of the 'left' image: noise and individual camera sensitivity alter pixel values (i.e. character substitutions); and different view angle reveals previously occluded data and introduces new occlusions (i.e. insertion and deletion of characters). Consequently, the $\mathrm{N} \& \mathrm{~W}$ approach is a very good starting point for developing a stereo matching algorithm, as seen in (MacLean, et al., 2010). The novelty of this work is that, first, it takes full advantage of the protein sequence/scanline analogy by refining the $\mathrm{N} \& \mathrm{~W}$ based stereo matching algorithm with the relevant extensions proposed in the field of bioinformatics. Secondly, this analogy is exploited further by producing a graph which suggests the limits of applicability of the algorithm in term of image overlap and pixel occlusion.

\subsubsection{Scoring matrix}

Scoring matrices are filled in using scoring functions which quantify the cost of possible mutations. Different substitutions in protein sequences affect differently protein functions. However, this is not reflected in the match/mismatch dichotomy used in $\mathrm{N} \& \mathrm{~W}$. This was addressed by customising mismatch costs according to estimated rates of mutations between pairs of characters (Dayhoff, 1978) and (Henikoff \& Henikoff 1992). Although it would be possible to perform a statistical study to establish the mutation frequency between pixel values, here we use a linear model which is context independent. The mismatch penalty of aligning a pair of pixels, where $p_{i}$ and $p_{j}$ are their values, is expressed by the absolute value of their difference, so that extending an alignment along the diagonal alters the global score by:

$$
\text { match }-\left|p_{i}-p_{j}\right|
$$

In genetics, 'indels' are rare and dramatic events which usually have negative effect on protein functions. Although the $\mathrm{N} \& \mathrm{~W}$ can penalise this type of mutations by associating them with a higher cost than substitutions, it does not take into account that an 'indel' of $n$ characters is much more likely than $n$ 'indels' of one character. For this reason, the initial scoring scheme was completed with a lower penalty for extended gaps, egap, which encourages gaps to cluster. We believe this concept is also valid in stereo matching where one would expect that a few occlusions of several-pixel length would be more frequent than a large number of 1-pixel occlusions: due to the nature of stereo matching, different camera viewpoints create occlusion areas associated with each object present in a scene. Accordingly, we implemented extended gaps in our algorithm.

As a consequence of these changes, our scoring matrix is filled in using the following pseudo-code: 


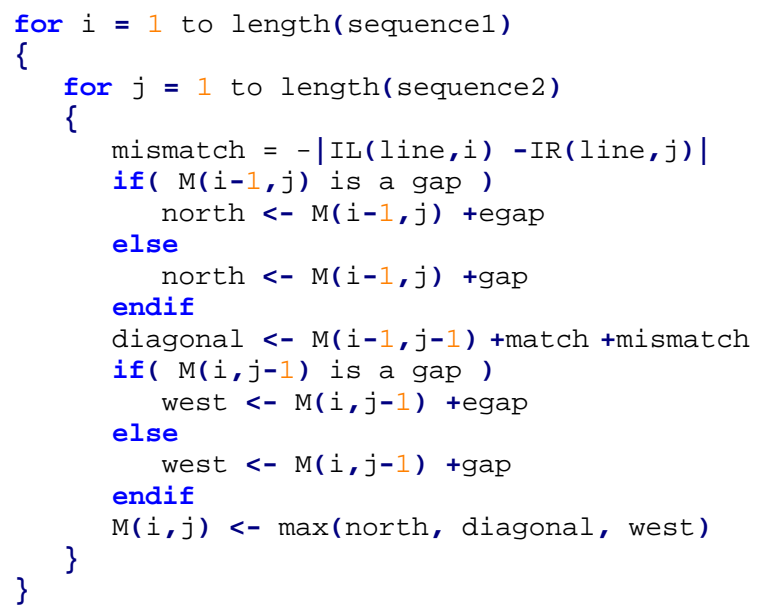

\subsubsection{Backtracking}

The N\&W backtracking process is straight forward. In the matrix, the cell with the highest score in either the last column or the last line of the matrix is identified. Then from that cell to the origin of the matrix, the global alignment is extracted using the stored direction information associated with each cell. This process usually produces a set of optimal alignments, see Fig. 1. Consequently, new information needs to be supplied to allow selecting a single solution. In bioinformatics, this is usually resolved by providing additional alignments involving other related sequences. They are used to produce a single multiple alignment which optimises all pair-wise alignment constraints (Higgins, et al., 1994), (Notredame, et al. 2000), (Edgar, 2004) and (Lassmann \& Sonnhammer 2005).

Several strategies have been offered to deal with this issue in the context of stereo matching. Many suggest selecting the 'smoothest' solution in term of horizontal and vertical discontinuities along and across scanlines (Cox, et al., 1996) and (Bobick \& Intille 1999). Some are based on high confidence matches, such as edge intersections, which are identified during a pre-processing phase. These good matches are exploited as extra constraints in the choice of a unique solution (Bobick \& Intille 1999) and (Torr \& Criminisi 2004).

In this work, we follow the traditional bioinformatics approach. The general principle is that each scanline can be seen as a mutation of both the previous and the following lines. Therefore, alignments involving these lines can be used to select among several solutions by enforcing some vertical discontinuities.

However, this approach is only valid if those lines are, indeed, mutations of the scanline of interest. Since usage of a neighbouring line does not ensure that the scanlines are related - there may be a horizontal edge -, we impose that the pixel sequences used as constraints are composed of the mean values between the scanline and neighbouring line pixels. For instance, in addition to the alignment between the scanline, $i$, on the left image $\left(l_{i}\right)$ and its corresponding line on the right one $\left(\mathrm{r}_{\mathrm{i}}\right)$, we can calculate a constraining alignment between $l_{i}$ and the average between $r_{i}$ and $r_{i+1}$. Those solutions present in both alignments are more likely to be a correct solution. Following this reasoning, using all combinations between $\left(l_{i}\right), \quad\left(l_{i}+l_{i+1}\right), \quad\left(l_{i-1}+l_{i}\right), \quad\left(l_{i}-\right.$ $\left.{ }_{1}+l_{i}+l_{i+1}\right)$, and $\left(r_{i}\right),\left(r_{i}+r_{i+1}\right),\left(r_{i-1}+r_{i}\right),\left(r_{i-1}+r_{i}+r_{i+1}\right)$, and reading scanlines from right to left, a total number of 32 constraints are generated.

In practice, in order to reduce the added computational cost of this strategy, we generate only constraining alignments for the subsections of scanline pairs which display several optimal correspondences, see Fig. 1. Then, for a given subsection, each initial solution is scored according to the number of constraining alignments which reach the same solution. The solution with the highest number of votes is selected. In the case of a draw, it is chosen at random.

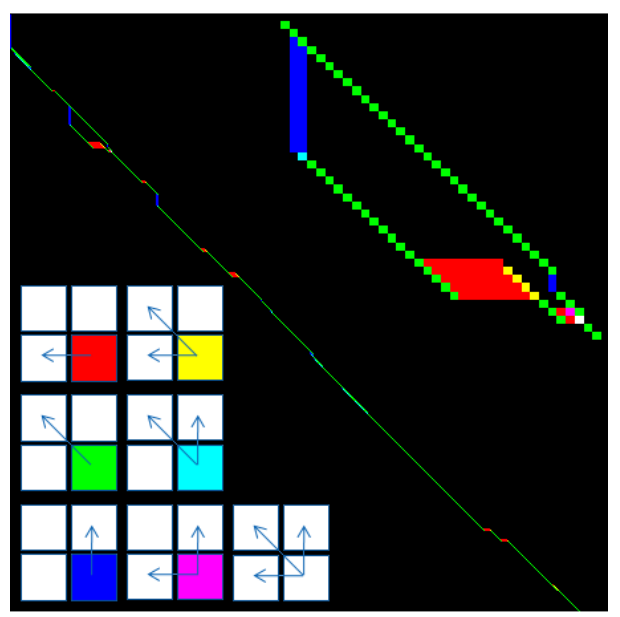

Figure 1: Paths returned for a pair of scanlines. Colours legend given in the bottom left- show valid direction(s) which can be followed to produce an optimal alignment. Zoom on an area with alternative paths is provided in the top right of the figure.

\subsubsection{Scope}

Traditional stereo matching algorithms are applied on rectified pair of images which share a 'sufficient' amount of overlap. To our knowledge, no statistically reliable study has quantified that 
amount. Since we use a bioinformatics-inspired framework, we propose to get an insight about this by investigating the confidence which is given to protein alignments according to the amounts of overlap and mutations.

Due to the availability of protein sequences, e.g. more than 20 million entries in UniProt (Leinonen, et al., 2004), and its usage in major international projects such as the Human Genome Project (International Human Genome Sequencing Consortium, 2001), the validity of sequence alignments have been the subject of statistical and experimental studies (Karlin \& Altschul 1990), (Pearson, 1998) and (Rost, 1999). Those statistics were integrated in the main sequence alignment servers (Altschul, et al., 1997) and (Mackey, et al., 2002). The outcome of these studies can be illustrated by the graph produced by Rost (Rost, 1999), where alignment meaningfulness is expressed according to the number of characters which can be aligned (i.e. overlap) and the percentage of characters with identical values (see Fig. 2).

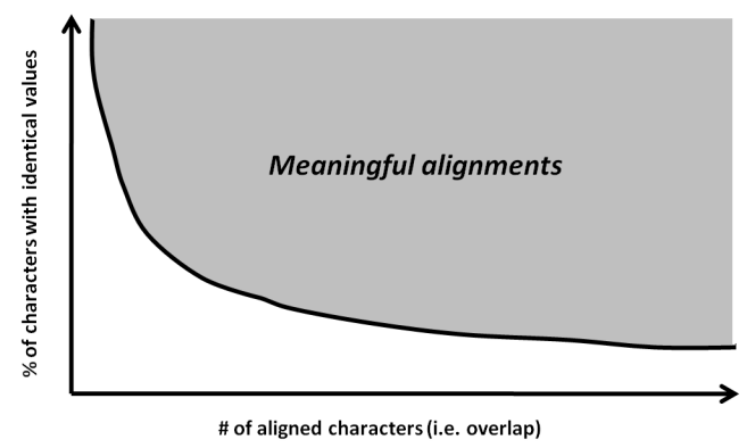

Figure 2: Expression of protein alignment meaningfulness according to the number of characters which can be aligned (i.e. overlap) and the percentage of characters with identical values, adapted from (Rost 1999)

In this paper, we propose to produce such a curve for stereo pair images and infer from it if alignments are meaningful.

\section{RESULTS}

\subsection{Experimental setup}

In order to validate our algorithm, experiments are conducted using the benchmarking framework from Middlebury (Scharstei et al. 2002-2003), which has been largely accepted by the computer vision community for objective comparison of stereo matching algorithms.
When several optimal solutions are returned by our algorithm, the selection of a unique solution is achieved by applying the 32 different constraints imposing scanline continuity. Finally, when specified, disparity maps are post-processed by a median filter (MF), which has been shown as a powerful and simple way to improve results (Mühlmann, et al., 2002).

\subsubsection{Automatic parameter configuration}

First score parameters, i.e. match, gap and extended gap, must be configured. Since they are sensitive to image characteristics, they need to be customised for each stereo pair. If the actual disparity map is known, an optimisation function can be applied to maximise matching accuracy. Although such process allows evaluating the best possible performance of an algorithm, it does not have practical applications.

Therefore, a methodology for automatic parameter configuration is required. We propose to generate a pseudo ground truth disparity map by establishing sparse pixel correspondences using the SIFT algorithm (Local Invariant Feature Transform). Key points on corresponding scanlines are paired and used to calculate disparity values (code available at www.cs.ubc.ca/ lowe/keypoints/). However, since only a few pairs are detected (fewer than 100 for images from the Middlebury dataset), this initial set is not suitable for parameter optimisation. In order to increase this number, Delaunay triangulation is applied to estimate the disparity of more pixels (see Fig. 3). Using the key points as vertices, homographic transformations between both meshes can be calculated for each triangle. These transformations are then used to estimate a larger set of disparity values. Obviously, this approximation can only be considered as valid for small triangles: in this work, only triangles whose sides are shorter than 15 pixels are used. As Table 3 shows, those disparity values are very accurate since they have an average pixel error below 0.5 . Consequently, such disparity maps can be considered as ground truth and used for parameter optimisation.

Table 3: Automatic ground truth generated for parameter configuration and its estimated error.

\begin{tabular}{|c|c|c|}
\cline { 2 - 3 } \multicolumn{1}{c|}{} & $\begin{array}{c}\text { \# of disparity } \\
\text { points }\end{array}$ & $\begin{array}{c}\text { Average } \\
\text { error }\end{array}$ \\
\hline Cones & 657 & 0.36 \\
\hline Teddy & 190 & 0.46 \\
\hline Venus & 211 & 0.27 \\
\hline Tsukuba & 600 & 0.45 \\
\hline
\end{tabular}




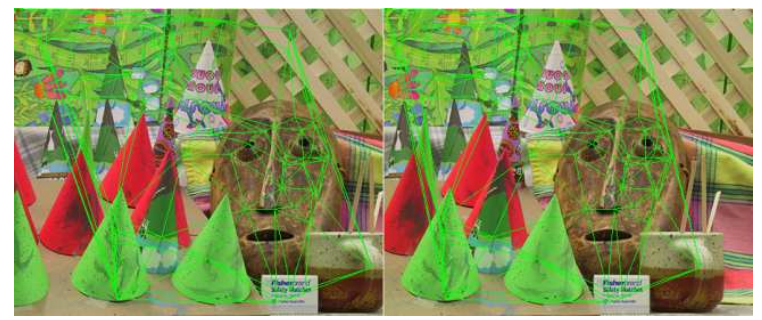

Figure 3: Delaunay triangulation of left and right images using the generated pairs of key points.

\subsection{Performances}

\subsubsection{Stereo-pair correspondences}

Fig. 4 shows raw disparity maps, i.e. without smoothing, obtained for the images used in the Middlebury framework. In Table 4, quantitative results are provided to validate our algorithm. The table quantifies the introduction of extended gaps (EG) and the procedure for automatic parameter selection (AP). In addition performances are provided for state of the art methods: the reference for scanline-based DP (Bobick \& Intille 1999), treebased DP which addresses inter-scanline coherence in the optimisation process (Veksler, 2005) and (Deng \& Lin 2006), and a segment-based stereo matcher (Klaus et al. 2006).

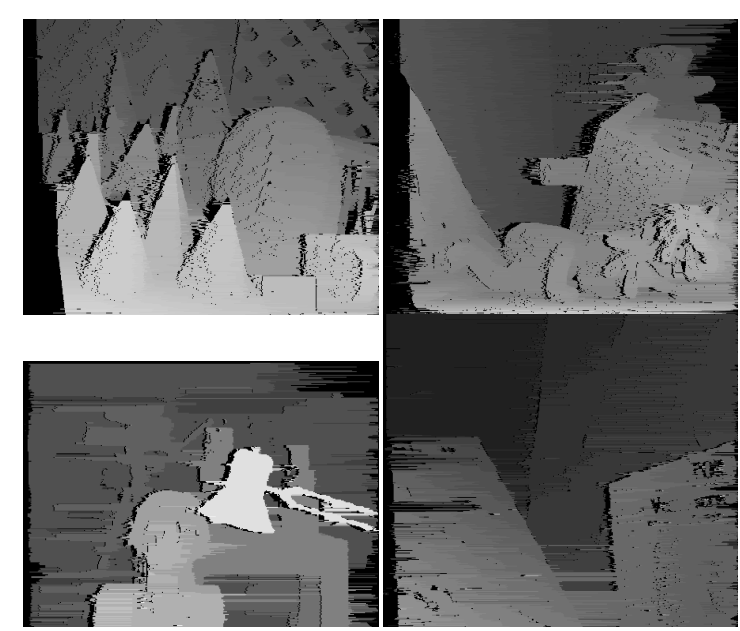

Figure 4: Disparity maps.

When optimising parameters using the ground truth disparity map as other methods do, in its most advanced configuration (i.e. our approach +EG $+\mathrm{MF}$ ) the proposed framework outperforms Bobick's (Bobick \& Intille 1999). Although, in terms of accuracy it cannot compete with the more computationally expensive approaches, ours could operate in real time as shown by (MacLean, et al., 2010).

Analysis of Table 4 confirms that the inclusion of extended gaps suits the nature of occlusions since performances are significantly improved. Moreover, as expected, the application of a median filter on disparity maps, which introduces some interscanline coherence, increases accuracy. Finally, results obtained using the AP configuration demonstrates that, although performances are degraded compared to those produced by a system using optimal parameters, they are still satisfactory. For example, our approach outperforms Bobick's (Bobick \& Intille 1999) in 3 image pairs out of 4.

Table 4: Performance comparison. EG: with extended gaps

AP: with automatic parameter selection MF: with median filter

\begin{tabular}{|c|c|c|c|c|c|}
\hline$\%$ & $\begin{array}{c}\text { Tsukuba } \\
\text { (non occ) }\end{array}$ & $\begin{array}{c}\text { Venus } \\
\text { (non occ) }\end{array}$ & $\begin{array}{c}\text { Teddy } \\
\text { (non occ) }\end{array}$ & $\begin{array}{c}\text { Cones } \\
\text { (non } \\
\text { occ) }\end{array}$ & $\begin{array}{c}\text { All } \\
\text { (bad } \\
\text { pixels) }\end{array}$ \\
\hline $\begin{array}{c}\text { Klaus et } \\
\text { al. 2006 }\end{array}$ & 1.11 & 0.11 & 4.22 & 2.48 & 4.23 \\
\hline $\begin{array}{c}\text { Veksler } \\
\text { 2005 }\end{array}$ & 1.99 & 1.41 & 15.9 & 10.0 & 11.7 \\
\hline $\begin{array}{c}\text { Deng \& } \\
\text { Lin 2006 }\end{array}$ & 2.21 & 0.46 & 9.58 & 3.23 & 6.82 \\
\hline $\begin{array}{c}\text { Bobick \& } \\
\text { Intille } \\
1999\end{array}$ & 4.12 & 10.1 & 14.0 & 10.5 & 14.2 \\
\hline $\begin{array}{c}\text { Our } \\
\text { approach }\end{array}$ & 6.67 & 12.0 & 15.5 & 12.7 & 18.6 \\
\hline $\begin{array}{c}\text { Our } \\
\text { approach } \\
\text { +EG }\end{array}$ & 6.74 & 10.7 & 14.1 & 11.0 & 16.7 \\
\hline $\begin{array}{c}\text { Our } \\
\text { approach } \\
\text { +EG } \\
\text { +MF }\end{array}$ & 4.63 & 7.40 & 10.7 & 7.75 & 13.4 \\
\hline $\begin{array}{c}\text { Our } \\
\text { approach } \\
\text { +AP +EG } \\
\text { +MF }\end{array}$ & 7.61 & 7.87 & 10.8 & 8.59 & 14.9 \\
\hline
\end{tabular}

These results confirm the validity of the analogy made between stereo matching and protein sequence alignment.

\subsubsection{Stereo matching meaningfulness}

Following the efforts of Rost (Rost 1999) who experimentally defined a curve under which protein 
alignment may become meaningless (see Fig. 2), we have produced a similar graph plotting accuracy as a function of image overlap and pixel similarity to express the meaningfulness of matching an image pair.

In this section, we consider that the alignment between two scanlines $\mathrm{L}$ and $\mathrm{R}$ is meaningful if the optimal score obtained for this alignment according to the scoring matrix is the highest score that $\mathrm{L}$ can achieved against any scanline of the right image.

In the original paper by Rost, results were plotted in a graph showing the number of residues aligned versus the percentage of character similarity. In our particular case, we can assimilate the number of aligned characters as the number of pixels matched between the images, or equivalently, the percentage of overlap between the images. Regarding the second axis, it can be understood as the percentage of identical pixels existing between both images.

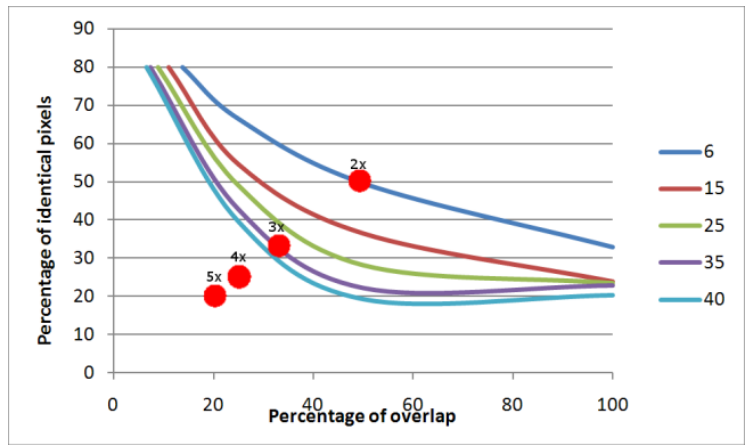

Figure 5: Scanline correspondence errors according to overlap and pixel similarity. Red dots show where matching experiments between an image and its zoomed version (from $2 \mathrm{x}$ to $5 \mathrm{x}$ ) would fit on this graph.

Unlike in bioinformatics, pixel value changes have a continuous nature, Consequently, the estimation of pixel similarity between a pair of stereo images would depend on a threshold. In order to have an absolute control of all variables, the conditions of the matching experiments were simplified. Our algorithm was applied to match an image with a tranformed version of itself. Pixel identity was controlled by adding 'salt and pepper' to the image, while the percentage of overlap was simulated by removing the required number of pixel columns. On Fig. 5, points are connected according to the measure accuracy in terms of percentage of scanlines matching the correct scanlines (see Fig. 6). Thus, a set of curves were created highlighting the image pair characteristics required to obtain matching errors between $6 \%$ and $40 \%$. As expected the shape of these curves is very similar to Rost's (Fig. 2), which reinforces the value of our analogy between stereo matching and protein sequence alignment. As in Rost's case, we believe the graph in Fig. 5 can estimate the meaningfulness of stereo correspondences using image overlap and the number of identical pixels as parameters.

In order to test this hypothesis, we propose to predict the outcome of matching image pairs captured at different resolution. On Fig. 5, we have plotted in red the estimated overlap and pixel similarity between images where the second one was taken with a zoom of $2 x, 3 x, 4 x$ and $5 x$. Using the predictive accuracy suggested by the curve set, one can infer that matching with $2 \mathrm{x}$ or $3 \mathrm{x}$ image should provide meaningful results, whereas results obtained for pairs including a $4 \mathrm{x}$ or $5 \mathrm{x}$ zoomed image should be meaningless. These predictions are tested in the next section.

\subsubsection{Matching images captured at different resolutions}

First, to evaluate the predictions made from the meaningfulness graph, the 'cone' image and its zoomed versions were processed using our algorithm. Here, the extended gap feature is deactivated since the assumption it is based on is not valid when dealing with images captured at different resolutions. Each scanline of the standard image was aligned against all scanlines of the zoomed image. The alignment with the highest score in the scoring matrix is then automatically selected to determine matching scanline pairs.

Fig. 7 shows the results of this procedure for different zoom values. As expected, the performances worsen with zoom increase. In the case of $\mathrm{x} 2$ and $\mathrm{x} 3$ zooms, correspondences between lines are usually correct or shifted by only a few lines, correlations between actual and predicted corresponding scanlines are 0.997 and 0.643 respectively. However, matching using $\mathrm{x} 4$ and $\mathrm{x} 5$ zooms produce associations which are often meaningless as quantified by the calculated correlations, i.e. 0.495 and 0.209 respectively. These experimental results confirm the predictions formulated using the meaningfulness graph.

Although finding meaningful correspondences between lines is essential for accurate stereo matching, it is not sufficient to assess the quality of pixel matching itself. Consequently, we undertook a second experiment where we calculated pixel correspondences between the left and the right images of the 'cone' stereo pair, where the right 
image was subject to a zoom transformation. Here, we assume that line pairings between the left and right images are known. In practice, if dealing with static cameras, this can be calculated if the zoom ratio is known. Otherwise, the procedure described in the previous experiment can be used to initialise a line pairing optimisation algorithm. Colour images in Fig. 6 show pairs of images, i.e. a) and b) where the lines of the left image were removed so that both images have the same number of scanlines. Images c) and d) display calculated and actual disparity maps. In agreement with the previous experiment, only usage of $\mathrm{x} 2$ and $\mathrm{x} 3$ zooms produces meaningful results, even if, in the case of $\mathrm{x} 3$ zoom experiment, the accuracy is poor.

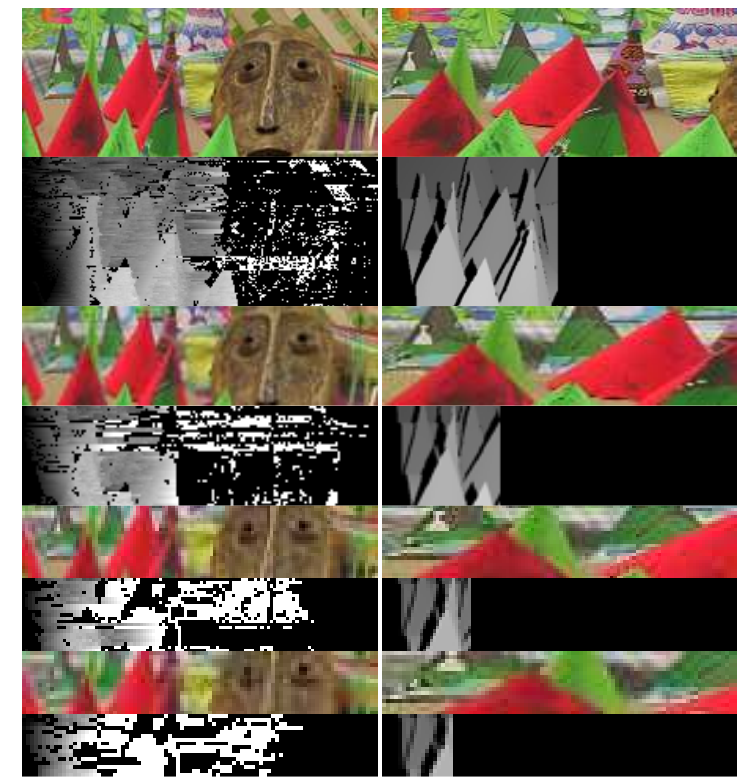

Figure 6: a) Left and b) right images used for zoom $x 2, x 3$, $\mathrm{x} 4$ and $\mathrm{x} 5, \mathrm{c})$ corresponding computed and d) ground truth disparity maps

\section{Conclusions}

The main contribution of this paper resides in the exploitation of the analogy between protein sequence alignment and image pair correspondence to design a bioinformatics-inspired framework for stereo matching based on dynamic programming. Not only does this approach offer accurate results with an algorithm which is suitable for real-time implementation, but it motivated the creation of a meaningfulness graph, which helps to predict matching validity according to image overlap and pixel similarity. This graph led to the application of the proposed method to the unexplored scenario of stereo matching between images captured at different resolutions. Experimental results reveal that good performance can be obtained when there is a ratio of 2 between the resolutions of a pair of images.

Another contribution of this work is an automatic procedure to estimate automatically all matching parameters. Conducted experiments showed that high quality sparse pixel correspondences generated by the SIFT algorithm could be extended using a Delaunay triangulation approach to generate a partial disparity map suitable for parameter optimisation.

In future work, we intend to exploit further the computer vision/bioinformatics analogy by considering video frames as leaves of a phylogenetic tree.
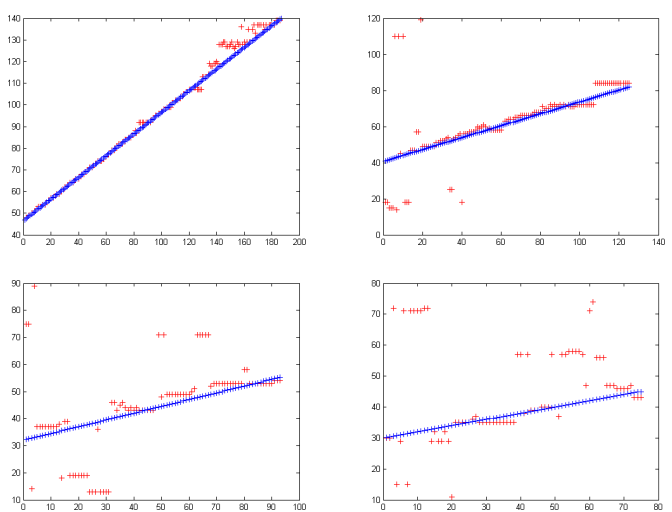

Figure 7: Correspondences between lines on the standard and zoomed images for zooms of a) $2 x, b) 3 x, c) 4 x$ and d) $5 \mathrm{x}$ : calculated (red) and ground truth (blue).

\section{REFERENCES}

Altschul, S.F., Madden, T.L., Schäffer, A.A., Zhang, J., Zhang, Z., Miller, W., Lipman, D.J., 1997. Gapped BLAST and PSI-BLAST: a new generation of protein database search programs. Nucleic Acids Research, 25, pp.3389-3402.

Baker, H., Binford, T., 1981. Depth from edge and intensity based stereo. In IJCAI81, pp.631-636.

Belhumeur, P.N., 1996. A Bayesian approach to binocular stereopsis. International Journal of Computer Vision, 19(3), pp.237-260.

Bobick, A.F., Intille, S.S., 1999. Large occlusion stereo. International Journal of Computer Vision, 33(3), pp.181-200.

Cox, I.J., Hingorani, S.L., Rao, S.B., Maggs, B. M., 1996. A maximum likelihood stereo algorithm. Computer Vision and Image Understanding, 63(3), pp.542-567.

Dayhoff, M.O., Eck, R.V., Chang, M.A., Sochard, M.R., 1965. Atlas of Protein Sequence and Structure 1965, National Biomedical Research Foundation, Silver Spring, Maryland. 
Dayhoff, M.O., 1978. Atlas of Protein Sequence and Structure, Suppl 3, National Biomedical Research Foundation, Silver Spring, Maryland.

Deng, Y., Lin, X., 2006. A fast line segment based dense stereo algorithm using tree dynamic programming. In European Conference on Computer Vision, Graz, Austria, May 7 - 13, 2006.

Edgar, R.C., 2004. MUSCLE: a multiple sequence alignment method with reduced time and space complexity. BMC Bioinformatics, 5, pp.113.

Forstmann, S., Kanou, Y., Ohya, J., Thuering, S., Schmitt, A., 2004. Real-Time Stereo by using Dynamic Programming, In Computer Vision and Pattern Recognition Workshop, Washington, DC, USA, 27 June-2 July 2004.

Geiger, D., Ladendorf, B., Yuille, A., 1992. Occlusions and binocular stereo. In European Conference on Computer Vision, pp.425-433.

Henikoff, S., Henikoff, J., 1992. Amino acid substitution matrices from protein blocks. Proceedings of the National Academy of Sciences, 89, pp.10915-10919.

Higgins, D., Thompson, J., Gibson, T., Thompson, J.D., Higgins, D.G., Gibson, T.J., 1994. CLUSTAL W: improving the sensitivity of progressive multiple sequence alignment through sequence weighting, position-specific gap penalties and weight matrix choice. Nucleic Acids Research, 22, pp.4673-4680.

International Human Genome Sequencing Consortium, 2001. Initial sequencing and analysis of the human genome. Nature, 409, pp.860-921.

Karlin, S., Altschul, S.F., 1990. Methods for assessing the statistical significance of molecular sequence features by using general scoring schemes. Proceedings of the National Academy of Sciences, 87, pp.2264-2268.

Klaus, A, Sormann, M, Karner, K, Segment-based stereo matching using belief propagation and a self-adapting dissimilarity measure, ICPR 3, pp. 15-18.

Lassmann, T., Sonnhammer, E.L.L., 2005. Kalign - an accurate and fast multiple sequence alignment algorithm. BMC Bioinformatics, 6, pp.298.

Lazaros, N., Sirakoulis, G.C., Gasteratos A., 2008. Review of Stereo Vision Algorithms: From Software to Hardware. International Journal of Optomechatronics, 2(4), pp.435 - 462.

Leinonen, R., Diez, F.G., Binns, D., Fleischmann, W., Lopez, R., Apweiler, R., 2004. UniProt Archive. Bioinformatics, 20, pp.3236-3237.

Mackey, A.J., Haystead, T.A., Pearson, W.R., 2002. Getting more from less: algorithms for rapid protein identification with multiple short peptide sequences. Molecular and Cellular Proteomics, 1(2), pp.139-147.
MacLean, W.J., Sabihuddin, S., Islam, J., 2010. Leveraging cost matrix structure for hardware implementation of stereo disparity computation using dynamic programming. Computer Vision and Image Understanding, In Press.

Mühlmann, K., Maier, D., Hesser, J., Männer, R. 2002. Calculating Dense Disparity Maps From Color Stereo Images, An Efficient Implementation. International Journal of Computer Vision, 47(3), pp.78-88.

Needleman, S.B., Wunsch, C.D., 1970. A general method applicable to the search for similarities in the amino acid sequence of two proteins. Journal of Molecular Biology, 48(3), pp.443-53.

Notredame, C., Higgins, D., Heringa, J., 2000. T-Coffee: A novel method for multiple sequence alignments. Journal of Molecular Biology, 302, pp.205-217.

Ohta, Y., Kanade, T., 1985. Stereo by intra- and interscanline search using dynamic programming. IEEE TPAMI, 7(2), pp.139-154.

Pearson, W.R., 1998. Empirical statistical estimates for sequence similarity searches. Journal of Molecular Biology, 276, pp.71-84.

Rost, B., 1999. Twilight zone of protein sequence alignments. Protein Engineering. 12(2), pp.85-94.

Salmen, J., Schlipsing, M., Edelbrunner, J., Hegemann, S., Lueke, S., 2009. Real-time stereo vision: making more out of dynamic programming. In International Conference on Computer Analysis of Images and Patterns, Münster, Germany, Sept. 2-4, 2009.

Scharstein, D., Szeliski, R, 2002. A taxonomy and evaluation of dense two-frame stereo correspondence algorithms. International Journal of Computer Vision, 47(1), pp.7-42.

Scharstein, D., Szeliski, R, 2003. High-accuracy stereo depth maps using structured light. In IEEE Computer Society Conference on Computer Vision and Pattern Recognition (CVPR 2003), vol. 1, pp. 195-202, June 2003.

Torr, P.H.S., Criminisi, A., 2004. Dense stereo using pivoted dynamic programming. Image and Vision Computing, 22(10), pp.795-806.

Veksler, O., 2005. Stereo correspondence by dynamic programming on a tree. In Computer Vision and Pattern Recognition, San Diego, CA, USA, 20-26 June 2005.

Wang, L., Liao, M., Gong, M., Yang, R., Nistér, D., 2006. High-quality real-time stereo using adaptive cost aggregation and dynamic programming. In $3 D$ Data Processing, Visualization and Transmission. Chapel Hill, USA, June 14-16, 2006. 